\title{
High-energy heavy-ions physics: from RHIC to LHC
}

\author{
David d'Enterria ${ }^{\mathrm{a}}$

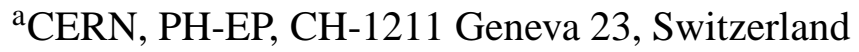

A selection of experimental results in high-energy nucleus-nucleus collisions after five years of operation of the Relativistic Heavy-Ion Collider (RHIC) is presented. Emphasis is put on measurements that provide direct information on fundamental properties of high-density QCD matter. The new experimental opportunities accessible at LHC are introduced, in particular those that may help clarify some of the current open issues at RHIC.

\section{Introduction}

Quantum Chromodynamics (QCD) is the only quantum field theory of the Standard Model whose collective behaviour (phase diagram and phase transitions) is accessible to study in the laboratory. High-energy heavy-ion (AA) collisions offer the only experimental means known so far to concentrate a significant amount of energy $(\mathscr{O}(1,10 \mathrm{TeV})$ at RHIC,LHC) in a "large" volume $\left(\mathscr{O}\left(100 \mathrm{fm}^{3}\right)\right.$ at thermalization times of $\left.\tau_{0} \approx 1 \mathrm{fm} / c\right)$. The study of the many-body dynamics of high-density QCD covers a vast range of fundamental physics problems:

- Deconfinement and chiral symmetry restoration: Lattice QCD calculations predict a new form of matter at energies densities above $\varepsilon \approx 1 \mathrm{GeV} / \mathrm{fm}^{3}$ consisting of an extended volume of deconfined and bare-mass quarks and gluons: the Quark Gluon Plasma (QGP) [ 1]. The scrutiny of this new state of matter (equation-of-state, order of the phase transition, ...) promises to shed light on fundamental questions of the strong interaction such as the nature of confinement, the mechanism of mass generation (chiral symmetry breaking, structure of the QCD vacuum) and hadronization, which still evade a thorough theoretical description due to their highly non-perturbative nature [2].

- Early universe cosmology: The quark-hadron phase transition took place some $10 \mu \mathrm{s}$ after the Big-Bang and was the most important event taking place in the Universe between the electro-weak (or SUSY) transition $\left(\tau \sim 10^{-10} \mathrm{~s}\right)$ and Big Bang nucleosynthesis ( $\tau \sim 200 \mathrm{~s}$ ). Depending on the order of the QCD phase transition ${ }^{1}$, several cosmological implications such as the formation of strangelets and cold dark-matter (WIMP) clumps or baryon fluctuations leading to inhomogeneous nucleosynthesis, have been postulated [ 3].

- Parton structure and evolution at small- $x$ : At high energies, hadrons consist of a very dense system of gluons with small (Bjorken) parton fractional momenta $x=p_{\text {parton }} / p_{\text {hadron }}$.

\footnotetext{
${ }^{1}$ The order itself is not exactly known: the pure SU(3) gauge theory is first-order whereas introduction of $2+1$ flavours makes it of a fast cross-over type [1].
} 
At low- $x$, the probability to emit an extra gluon is large $\propto \alpha_{s} \ln (1 / x)$ and non-linear $g g$ processes will eventually dominate the parton evolution in the hadronic wave functions. Whereas HERA results indicate that for $x \gtrsim 10^{-3}$, the parton evolution with $Q^{2}$ (or $\ln (1 / x)$ ) is described by the usual DGLAP (or BFKL) equations, at lower values of $x$ and around a saturation momentum of $Q_{s}^{2} \sim 2 \mathrm{GeV}^{2}$, such a configuration is theoretically described in terms of the "Colour Glass Condensate" (CGC) picture [4]. Since the nonlinear growth of the gluon density depends on the transverse size of the system (i.e. $Q_{S}^{2} \propto A^{1 / 3}$, where $A$ is the number of nucleons in the nucleus), the effects of gluon saturation are expected to set in earlier for ultrarelativistic heavy nuclei than free nucleons.

- Gauge/String duality: Theoretical calculations based on the Anti-de-Sitter/ConformalField-Theory (AdS/CFT) correspondence permit to obtain results in strongly coupled $\left(\lambda=g^{2} N_{c} \gg 1\right) \mathrm{SU}\left(N_{c}\right)$ gauge theories in terms of a weakly-coupled dual gravity theory [ 5]. Recent applications of this formalism for QCD-like $\mathscr{N}=4$ super Yang-Mills theories have allowed to compute transport properties of experimental relevance, - such as the QGP viscosity [ 6], the "jet quenching" parameter $\langle\hat{q}\rangle$ [ 7], or the heavy-quark diffusion coefficient [ 8] -, from black hole thermodynamics calculations. Such results provide valuable insights on dynamical properties of non-perturbative QCD that cannot be directly treated by lattice methods.

In this overview, we present a selection of experimental results (mostly from the comprehensive reviews of the 4 RHIC experiments [ $9,10,11,12])$ from $\mathrm{AuAu}, \mathrm{dAu}$ and pp collisions up to a maximum center-of-mass energy of $\sqrt{s_{N N}}=200 \mathrm{GeV}$. Direct information on the thermodynamical and transport properties of the strongly interacting medium produced in AA collisions is commonly obtained by comparing the results for a given observable $\Phi_{A A}$ to those measured in $\mathrm{p}(\mathrm{d}) \mathrm{A}$ ("cold QCD matter") and in pp ("QCD vacuum") collisions as a function of center-ofmass energy, $p_{T}$, rapidity $y$, reaction centrality (impact parameter $b$ ), and particle type (mass):

$R_{A A}\left(\sqrt{s_{N N}}, p_{T}, y, m ; b\right)=\frac{\text { "hot/dense QCD medium" }}{\text { "QCD vacuum" }} \propto \frac{\Phi_{A A}\left(\sqrt{s_{N N}}, p_{T}, y, m ; b\right)}{\Phi_{p p}\left(\sqrt{s}, p_{T}, y, m\right)}$

The observed enhancements (e.g. in photon or baryon yields, or soft hadron slopes) and/or suppressions (e.g. in total multiplicities, high- $p_{T}$ leading hadrons, or quarkonia yields) in the $R_{A A}\left(\sqrt{s_{N N}}, p_{T}, y, m ; b\right)$ ratios can be directly related to the properties of the produced QCD matter after accounting for a realistic modeling of the space-time evolution of the collision process ${ }^{2}$.

\section{Reduced hadron multiplicities $\mapsto$ Saturated gluon distribution function $x G_{A}\left(x, Q^{2}\right)$ ?}

The bulk hadron multiplicities measured at mid-rapidity in central AuAu at $\sqrt{s_{N N}}=200 \mathrm{GeV}$ are $d N_{c h} /\left.d \eta\right|_{\eta=0} \approx 700$, comparatively lower than the $d N_{c h} /\left.d \eta\right|_{\eta=0} \approx 1000$ expectations [13] of "minijet" dominated scenarios, soft Regge models (without accounting for strong shadowing effects), or extrapolations from an incoherent sum of proton-proton collisions (Fig. 1, left). On the other hand, approaches [ 14, 15] based on gluon saturation [ 4], which take into account a reduced initial number of scattering centers in the nuclear parton distribution functions,

\footnotetext{
${ }^{2}$ The hot and dense systems produced in heavy-ion collisions at RHIC expand longitudinally (transversely) with $\langle\beta\rangle \approx 1.0(0.6)$ and stop self-interacting collectively at freeze-out times $\tau \approx 15 \mathrm{fm} / c$.
} 
$f_{a / A}\left(x, Q^{2}\right)<A \cdot f_{a / N}\left(x, Q^{2}\right)$, agree well with experimental data. In those CGC calculations, the final hadron multiplicities are assumed to be simply related to the initial number of released partons (local parton-hadron duality) which are depleted in the initial state compared to protonproton collisions due to non-linear gluon-gluon fusion effects.
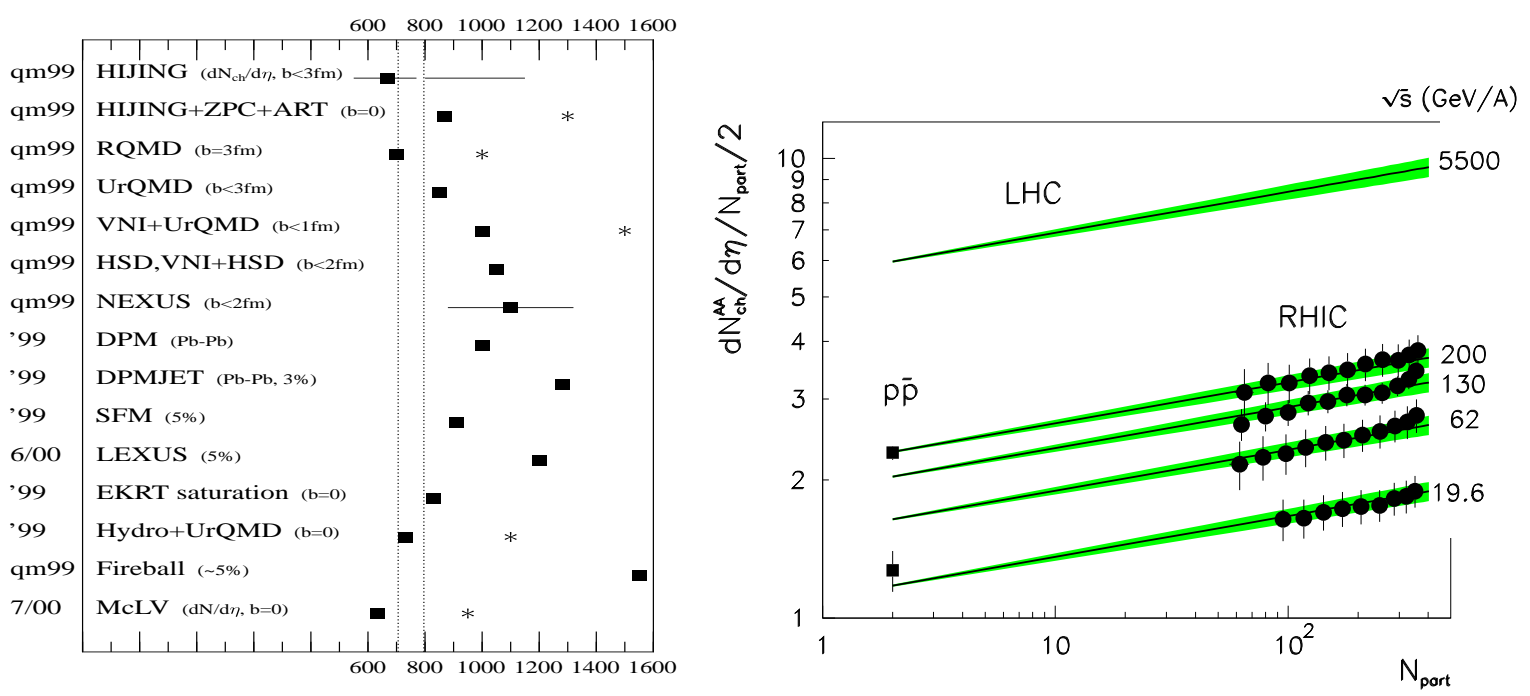

Figure 1. Left: Data versus models for $d N_{c h} /\left.d \eta\right|_{\eta=0}$ in AuAu at $\sqrt{s_{N N}}=200 \mathrm{GeV}$ [13]. Right: Energy and centrality dependences (in terms of the number of nucleons participating in the collision, $N_{\text {part }}$ ) of $d N_{c h} /\left.d \eta\right|_{\eta=0}$ (normalized by $N_{\text {part }}$ ): PHOBOS AuAu data [11] versus the predictions of the saturation approach [15] .

The good reproduction of the bulk AA hadron multiplicities (including its centrality and center-of-mass energy dependences, Fig. 1 right) has been one of the supporting arguments in favour of the existence of non-linear QCD effects in high-energy nuclear collisions. In addition, the BRAHMS observation of suppressed yields of moderately high- $p_{T}$ hadrons in dAu at forward rapidities $(\eta \approx 3.2)$ [ [12], as well as the "geometrical scaling"-like behaviour of the nuclear PDFs observed for $x<0.017$ [ [15], are also consistent with gluon saturation expectations. It is worth noting, however, that both results are in a kinematic range with relatively low momentum scales, $\mathscr{O}(1 \mathrm{GeV})$, where non-perturbative effects can blur a simple interpretation based on partonic degrees of freedom alone. Indeed, at RHIC (and HERA) energies the saturation momentum, - the scale at which non-linear effects become important and start to saturate the parton densities -, is in the transition between the soft and hard regimes $\left(Q_{s}^{2} \approx 2 \mathrm{GeV}^{2}\right)$. At LHC the relevance of low- $x$ QCD effects in hadronic collisions will be certainly enhanced due to the increased (i) center-of-mass energy $\sqrt{s_{N N}}$, (ii) nuclear radius $A^{1 / 3}$, and (iii) rapidity $y$ of the produced partons. Indeed, at LHC energies not only the relevant Bjorken $x$ values will be 30-70 times lower than at RHIC: $x_{2}^{\min }=\left(p_{T} / \sqrt{s_{N N}}\right) e^{-y} \approx 10^{-3}\left(10^{-5}\right)$ at central (forward) rapidities for processes with a hard scale $p_{T} \sim 10 \mathrm{GeV}$, but also the saturation momentum, $Q_{s}^{2} \sim A^{1 / 3} S_{N N}^{\lambda / 2} \approx 5-10 \mathrm{GeV}^{2}$ [14], will be in the perturbative range. 


\section{Strong radial and elliptic collective flows $\mapsto$ QGP as a perfect fluid ?}

The bulk of hadron production $\left(p_{T} \lesssim 2 \mathrm{GeV} / c\right)$ in AuAu at RHIC shows strong collective effects known as radial and elliptic flows. On the one hand, the measured single hadron $p_{T}$ spectra have an inverse slope parameter larger than that measured in pp collisions, increasing with reaction centrality and hadron mass as expected if collective expansion effects blue-shift the hadron spectra (Fig. 2] left). Phenomenological fits to "blast wave" models yield transverse flow velocities $\langle\beta\rangle \approx 0.6$ [ 9]. On the other hand, the azimuthal distribution $d N / d \phi$ of hadrons emitted w.r.t. the reaction plane show a strong harmonic modulation with a preferential "in-plane" emission in non-central collisions. Such an azimuthal flow pattern is a truly collective effect (absent in pp collisions) consistent with an efficient translation of the initial $x$-space anisotropy in non-central reactions (with an almond-shape overlap zone) into a final "elliptical" asymmetry in $p$-space. The amount of elliptic flow is quantified via the second Fourier coefficient $v_{2}=\langle\cos (2 \phi)\rangle_{p_{T}}$ of the $d N / d \phi$ distribution relative to the reaction plane. The large $v_{2} \approx 0.2$ measured in the data (Fig. 2] right) indicates a strong degree of collectivity (pressure gradients) developing in the first instants of the collision. Indeed, elliptic flow develops in the initial phase of the reaction and quickly self-quenches beyond $\tau \approx 5 \mathrm{fm} / c$ as the original spatial eccentricity disappears. This is confirmed by the observation that not only light hadrons but charm quarks (indirectly measured via the semileptonic decays of $\mathrm{D}$ mesons into $e^{ \pm}$) show a $v_{2}$ signal as large as $10 \%$ [17] clearly consistent with strong collective correlations during the partonic phase of the reaction.
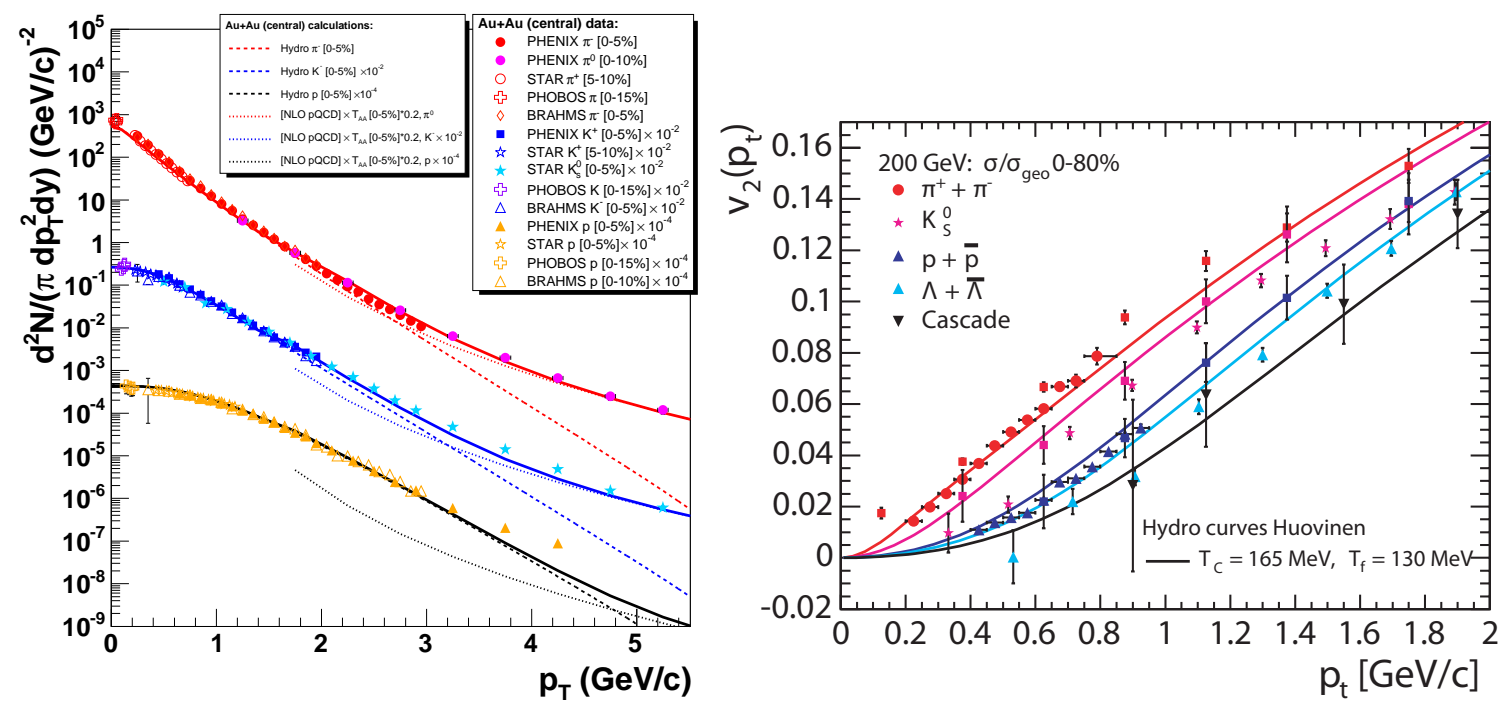

Figure 2. Left: Transverse spectra for pions, kaons, and protons measured below $p_{T} \approx 5$ $\mathrm{GeV} / \mathrm{c}$ in $0-10 \%$ most central AuAu collisions at $\sqrt{s_{N N}}=200 \mathrm{GeV}$, compared to hydrodynamics $(+\mathrm{pQCD})$ calculations [ [16]. Right: Measured elliptic flow parameter $v_{2}\left(p_{T}\right)$ for a variety of hadrons [18] compared to hydrodynamic predictions [19].

Interestingly, the strong $v_{2}$ seen in the data is inconsistent with the much lower values, $v_{2} \lesssim$ 
$6 \%$, expected for purely hadronic matter [ 20] as well as for a partonic system cascading with perturbative cross-sections $\left(\sigma_{g g} \approx 3 \mathrm{mb}\right)[21]$. The magnitude, $p_{T}$ and mass dependences of the radial and elliptic flows below $p_{T} \approx 2 \mathrm{GeV} / c$ are, however, remarkably well described by ideal hydrodynamics models whose space-time evolution starts with a QGP equation-of-state $($ EoS $)$ with initial $\varepsilon_{0} \approx 30 \mathrm{GeV} / \mathrm{fm}^{3}$ at very short thermalization times $\tau_{0} \approx 0.6 \mathrm{fm} / c$ [ 22, 23, 24, 19] (Fig. 2). The fast (local) thermalization times, the robust collective flow generated in the first instants of the reaction, and the excellent agreement of the data with ideal hydrodynamics models which assume a fluid evolution with zero viscosity, have been presented [ 25, 26, 27, 28, 29] as evidences that the QGP formed at RHIC is a strongly coupled liquid (characterized by Coulomb coupling parameter $\Gamma=\left\langle E_{\text {pot }}\right\rangle /\left\langle E_{\text {kin }}\right\rangle>1$ ) rather than a weakly interacting gas of partons. Estimates of the maximum amount of viscosity allowed by the $v_{2}\left(p_{T}\right)$ data [27] give a value close to the conjectured universal lower bound for the dimensionless viscosity/entropy ratio, $\eta / s=\hbar /(4 \pi)$, obtained from AdS/CFT calculations [6]. This result would make the QGP the most perfect fluid ever observed. The measurement of the differential elliptic flow properties in AA collisions at LHC will be of primary importance to confirm such an interpretation and search for a possible transition from a hotter weakly interacting QGP to the liquid-like state found at RHIC [26, 27].

\section{High $p_{T}$ hadron suppression $\mapsto$ dense QGP with $d N^{g} / d y \sim 1000,\langle\hat{q}\rangle \sim 14 \mathbf{G e V}^{2} / \mathbf{f m}$}

Among the most exciting results of the RHIC physics programme is the observed strong suppression of high- $p_{T}$ leading hadron spectra in central AA [ 30] consistent with the predicted energy loss of the parent light quarks and gluons traversing the dense colored medium ("jet quenching") [ 31]. Above $p_{T} \approx 5 \mathrm{GeV} / c, \pi^{0}, \eta$, and inclusive charged hadrons show all a common factor of $\sim 5$ suppression compared to an incoherent superposition of pp collisions (Fig. 3] left) [32]. The $R_{A A}=1$ perturbative expectation which holds for other hard probes such as "color blind" direct photons and for high- $p_{T}$ hadrons in dAu reactions (where no final-state dense and hot system is produced) [33], is badly broken $\left(R_{A A} \approx 0.2\right)$ in central AuAu collisions. Most of the empirical properties of the quenching factor for light-flavor hadrons (magnitude, $p_{T^{-}}$, centrality-, $\sqrt{s_{N N}}$ - dependences of the suppression) [ 34] are in quantitative agreement with the predictions of non-Abelian parton energy loss models which assume that the parent parton loses energy by gluonstrahlung while traversing a medium with a large color density. Very large initial gluon rapidity densities, $d N^{g} / d y \approx 1000$ [ 35], or equivalently, transport coefficients ${ }^{3}$, $\langle\hat{q}\rangle \approx 14 \mathrm{GeV}^{2} / \mathrm{fm}$ [ 37, 38], are needed to explain the amount of hadron suppression. However, the fact that the quenching factor for high- $p_{T}$ electrons from semi-leptonic $D$ and $B$ decays is as suppressed as the light hadrons in central AuAu (Fig. 3, right) [39, 40] is in apparent conflict with the robust $\Delta E_{Q}<\Delta E_{q}<\Delta E_{g}$ prediction of radiative energy loss models. In order to reproduce the high $p_{T}$ open charm/bottom suppression, jet quenching models require either initial gluon densities $\left(d N^{g} / d y \approx 3000\right)$ inconsistent with those needed to describe the quenched light hadron spectra [41, 42], or a smaller relative contribution of $B$ relative to $D$ mesons than theoretically expected in the measured decay electron $p_{T}$ range [ [38]. This discrepancy may point to an additional contribution from elastic (i.e. non-radiative) energy loss [ 43, 44] for heavyquarks [42] which was considered negligible so far. The unique possibility at LHC of fully reconstructing $c, b$ jets will be very valuable to clarify the response of strongly interacting mat-

\footnotetext{
${ }^{3}\langle\hat{q}\rangle$ characterizes the squared average $k_{T}$ transfer from the medium to the hard parton per unit distance [36].
} 

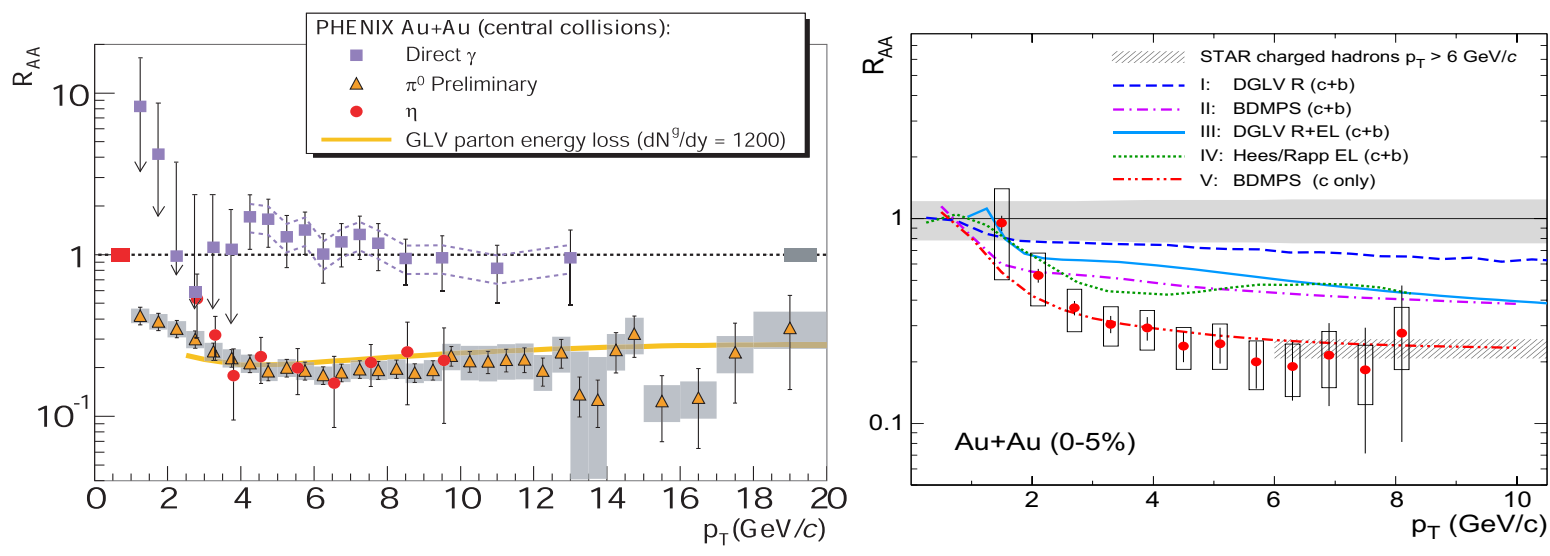

Figure 3. Nuclear modification factor $R_{A A}\left(p_{T}\right)$ for different particles measured in central $\mathrm{AuAu}$ at $\sqrt{s_{N N}}=200 \mathrm{GeV}$ compared to parton energy loss model predictions. Left: Direct $\gamma$, $\pi^{0}$, and $\eta$ [32]. Right: "Non-photonic" $e^{ \pm}$, mainly from D,B meson decays [ 40].

ter to fast heavy quarks, and provide detailed additional information on the transport properties of QCD matter [ [8].

\section{Modified semihard di-hadron $\phi$ correlations $\mapsto$ QGP speed of sound $c_{S}$ ?}

A second striking observation of the jet-quenching phenomena at RHIC is the strongly modified azimuthal dijet correlations compared to baseline pp results. Due to the difficulties of full jet reconstruction in AA at RHIC, jet-like correlations are measured on a statistical basis by selecting the highest $p_{T}$ trigger hadron of the event and measuring the azimuthal $(\Delta \phi=$ $\left.\phi-\phi_{\text {trig }}\right)$ and rapidity $\left(\Delta \eta=\eta-\eta_{\text {trig }}\right)$ distributions of associated hadrons $\left(p_{T, \text { assoc }}<p_{T, \text { trig }}\right)$ : $d^{2} N_{\text {pair }} / d \Delta \phi d \Delta \eta$. In pp collisions, a dijet signal appears as two distinct back-to-back Gaussian peaks at $\Delta \phi \approx 0$ (near-side) and at $\Delta \phi \approx \pi$ (away-side). At variance with this standard dijet topology in the QCD vacuum, the away-side dihadron azimuthal correlations $d N_{\text {pair }} / d \Delta \phi$ in central AuAu collisions shows a "dip" with a "double peak" structure at $\Delta \phi \approx \pi \pm 1.1$ (Fig. 4) for semihard associated hadrons $\left(p_{T, \text { assoc }}=1-2.5 \mathrm{GeV} / c\right)$ [ 45, 46]. Such a non-Gaussian ("volcano"-like) profile in the away-side hemisphere has been interpreted as due to the preferential emission of energy from the quenched parton at a finite angle with respect to the jet axis. Such a conical-like pattern can appear if the lost energy excites a collective mode of the medium and generates a wake of lower energy gluons with Mach- [47, 48] or Čerenkov-like [ 48, 49] angular emissions. In the first case, the speed of sound $d^{4}$ of the traversed matter, $c_{s}^{2}=\partial P / \partial \varepsilon$, can be determined from the characteristic supersonic angle of the emitted secondaries: $\cos \left(\theta_{M}\right)=c_{s}$, where $\theta_{M}$ is the Mach shock wave angle. The resulting preferential emission of secondary partons from the plasma measured at a fixed angle $\theta_{M} \approx 1.1$, yields an average value of the speed sound $c_{s} \approx 0.45$, larger than that of a hadron gas $\left(c_{s} \approx 0.35\right)$ [ [50], and not far from that of an ideal QGP $\left(c_{s}=1 / \sqrt{3}\right)$.

\footnotetext{
${ }^{4}$ The $c_{s}$ of an ultrarelativistic fluid is a simple proportionality constant relating its pressure and energy density: $P=c_{s}^{2} \varepsilon$.
} 

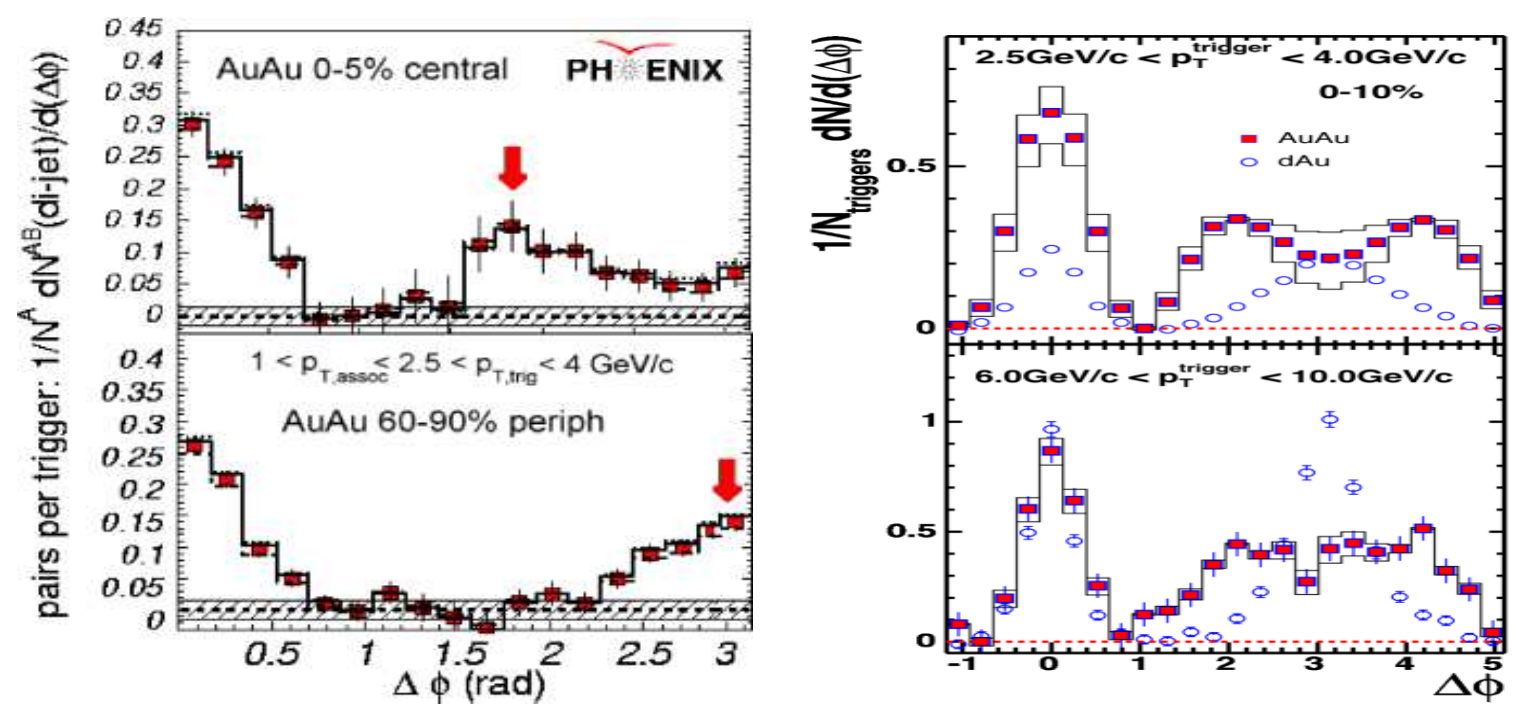

Figure 4. Azimuthal distributions of semihard hadrons ( $\left.p_{T, \text { assoc }}=1-2.5 \mathrm{GeV} / c\right)$ with respect to a trigger hadron measured at RHIC. Left: PHENIX data in central (top) and peripheral (bottom) AuAu [45]. Right: STAR data in central AuAu and dAu collisions [46].

\section{Summary}

A selection of experimental data from central AuAu collisions at RHIC energies $\left(\sqrt{s_{N N}}=\right.$ $200 \mathrm{GeV}$ ) has been presented providing direct information on fundamental thermodynamical and transport properties of high-density QCD matter. Four notable experimental results have been discussed: (i) the reduced total hadron multiplicities consistent with gluon saturation in the initial nuclear parton distribution functions, (ii) the strong transverse and elliptic differential flows in the bulk hadron spectra indicative of a high degree of collectivity and very low viscosities during the first instants of the reaction, (iii) the factor $\sim 5$ suppression of high $p_{T}$ leading hadrons reproduced by parton energy loss calculations for a medium with very large initial gluon densities $\left(d N^{g} / d y \approx 1000\right)$ and transport coefficient $\left(\langle\hat{q}\rangle \approx 14 \mathrm{GeV}^{2} / \mathrm{fm}\right)$, and (ii) the non-Gaussian shape of the azimuthal distributions of secondary hadrons in the away-side hemisphere of high- $p_{T}$ trigger hadrons attributed to Mach conical flow caused by the propagation of a supersonic parton through the dense system. Other interesting probes of quark-gluon matter (photons, quarkonia, ...), not covered here by lack of space, will be discussed in a coming publication [ 51]. Nucleus-nucleus collisions at LHC energies will undoubtedly contribute to expand the knowledge of many-body QCD at extreme conditions of temperature, density and low- $x$ shedding light on a vast ramification of fundamental physics problems.

\section{Acknowledgments}

This work is supported by the 6th EU Framework Programme contract MEIF-CT-2005-025073.

\section{REFERENCES}

1. See e.g. F. Karsch, Lect. Notes Phys. 583 (2002) 209.

2. A. M. Jaffe and E. Witten, "Quantum Yang-Mills Theory", Clay Maths Inst. Millennium 
Prize (2000), http://www.claymath.org/millennium/Yang-Mills_theory; and D. Gross, in “Ten Problems in Fundamental Physics" (2000), http://feynman.physics.1sa.umich.edu/strings2000/millennium.html.

3. D. J. Schwarz, Annalen Phys. 12 (2003) 220.

4. See e.g. E. Iancu and R. Venugopalan, in "QGP. Vol 3" Eds: R.C. Hwa and X.N. Wang, World Scientific, Singapore, hep-ph/0303204.

5. J. M. Maldacena, Adv. Theor. Math. Phys. 2 (1998) 231 [Int. J. Theor. Phys. 38 (1999) 1113]; E. Witten, Adv. Theor. Math. Phys. 2 (1998) 505.

6. P. Kovtun, D. T. Son and A. O. Starinets, Phys. Rev. Lett. 94 (2005) 111601.

7. H. Liu, K. Rajagopal and U. A. Wiedemann, hep-ph/0605178

8. J. Casalderrey-Solana and D. Teaney, hep-ph/0605199.

9. K. Adcox et al. [PHENIX], Nucl. Phys. A757 (2005) 184.

10. J. Adams et al. [STAR], Nucl. Phys. A757 (2005) 102.

11. B. B. Back et al. [PHOBOS], Nucl. Phys. A757 (2005) 28.

12. I. Arsene et al. [BRAHMS], Nucl. Phys. A757 (2005) 1.

13. K. J. Eskola, Nucl. Phys. A 698 (2002) 78.

14. D. Kharzeev and M. Nardi, Phys. Lett. B 507 (2001) 121; D. Kharzeev, E. Levin and M. Nardi, Nucl. Phys. A 747 (2005) 609.

15. N. Armesto, C. A. Salgado and U. A. Wiedemann, Phys. Rev. Lett. 94 (2005) 022002.

16. D. d'Enterria and D. Peressounko, Eur. Phys. J. C 46 (2006) 451

17. Y. Akiba [PHENIX], nucl-ex/0510008.

18. R. Snellings, AIP Conf. Proc. 756 (2005) 390.

19. P. Huovinen and P. V. Ruuskanen, Ann. Rev. Nucl. Part. Sci. to appear, nucl-th/0605008

20. X. 1. Zhu, M. Bleicher and H. Stoecker, Phys. Rev. C 72 (2005) 064911.

21. D. Molnar and M. Gyulassy, Nucl. Phys. A 697 (2002) 495 [Errat.-ibid. A 703 (2002) 893]

22. P. F. Kolb and U. Heinz, in "QGP. Vol 3" Eds: R.C. Hwa and X.N. Wang, World Scientific, Singapore, nucl-th/0305084, and refs. therein.

23. D. Teaney, J. Lauret and E. V. Shuryak, nucl-th/0110037.

24. T. Hirano and K. Tsuda, Phys. Rev. C 66 (2002) 054905.

25. E. Shuryak, Prog. Part. Nucl. Phys. 53 (2004) 273.

26. M. H. Thoma, J. Phys. G 31 (2005) L7.

27. T. Hirano and M. Gyulassy, Nucl. Phys. A 769 (2006) 71.

28. A. Peshier and W. Cassing, Phys. Rev. Lett. 94 (2005) 172301.

29. U. W. Heinz, nucl-th/0512051.

30. K.Adcox et al. [PHENIX], Phys. Rev. Lett. 88 (2002) 022301; S.Adler et al. [PHENIX], Phys. Rev. Lett. 91 (2003) 072301; J.Adams et al. [STAR], Phys. Rev. Lett. 91 (03) 172302.

31. M. Gyulassy, M. Plümer, Phys. Lett. B243 (1990) 432; X.N. Wang, M. Gyulassy, Phys. Rev. Lett. 68 (1992) 1480.

32. S. S. Adler et al. [PHENIX], Phys. Rev. Lett. 96 (2006) 202301; M. Shimomura [PHENIX], nucl-ex/0510023.

33. S. S. Adler et al. [PHENIX], Phys. Rev. Lett. 91 (2003) 072303; J. Adams et al. [STAR], Phys. Rev. Lett. 91 (2003) 072304.

34. D. d'Enterria, Eur. Phys. J. C43 (2005) 295.

35. I. Vitev and M. Gyulassy, Phys. Rev. Lett. 89 (2002) 252301.

36. R. Baier, Y. L. Dokshitzer, A. H. Mueller, S. Peigné and D. Schiff, Nucl. Phys. B484 (1997) 265; R. Baier, D. Schiff, B.G. Zakharov, Ann. Rev. Nucl. Part. Sci. 50 (2000) 37. 
37. A. Dainese, C. Loizides and G. Paic, Eur. Phys. J. C38 (2005) 461.

38. N. Armesto, A. Dainese, C. A. Salgado and U. A. Wiedemann, Phys. Rev. D71 (2005) 054027; N. Armesto et al., Phys. Lett. B 637 (2006) 362.

39. S. S. Adler et al. [PHENIX], Phys. Rev. Lett. 96 (2006) 032301

40. B. I. Abelev et al. [STAR], nucl-ex/0607012.

41. M. Djordjevic, M. Gyulassy and S. Wicks, Phys. Rev. Lett. 94 (2005) 112301.

42. S. Wicks, W. Horowitz, M. Djordjevic and M. Gyulassy, nucl-th/0512076.

43. M. G. Mustafa and M. H. Thoma, Acta Phys. Hung. A 22 (2005) 93.

44. A. Peshier, hep-ph/0607275.

45. S. S. Adler et al. [PHENIX], to appear in Phys. Rev. Lett. , nucl-ex/0507004.

46. T. Peitzmann [STAR], Proceeds. Hard Probes'06.

47. H. Stoecker, Nucl. Phys. A750, 121 (2005); J. Casalderrey, E. Shuryak, D. Teaney, J. Phys. Conf. Ser. 27 (2005) 22.

48. J. Ruppert and B. Muller, Phys. Lett. B618 (2005) 123.

49. V. Koch, A. Majumder and X. N. Wang, Phys. Rev. Lett. 96 (2006) 172302; I. M. Dremin, Nucl. Phys. A 767 (2006) 233.

50. B. Mohanty and J. e. Alam, Phys. Rev. C 68 (2003) 064903.

51. D. d'Enterria, in preparation. 